\title{
Controles colectivos de salud para niños: una experiencia en Villa Caracol, Bahía Blanca
}

\author{
Collective health checks for children: an experience in \\ Villa Caracol, Bahía Blanca
}

\author{
Méd. Paola Estefanía Buedoa, Lic. Claudia Guzmán ${ }^{a}$ Méd. Natalia Verónica Príncipe \\ Lic. Juliana Spinelli ${ }^{a}$ Lic. Susana Victoria Vollertsen ${ }^{a}$ y Mara Zabala ${ }^{a}$
}

\begin{abstract}
RESUMEN
Se presentan dificultades para lograr una cobertura efectiva de los controles de salud de los niños del barrio Villa Caracol de Bahía Blanca. El equipo de salud de la Unidad Sanitaria San Dionisio desarrolló un dispositivo denominado Controles Colectivos deSalud para Niños (CCSN). Los objetivos fueron reforzar el sentimiento de responsabilidad familiar respecto a la salud de los niños, realizar un tamizaje de niños que necesitaran un control de salud individual con urgencia y afianzar los espacios de vida personales con los que contaba cada sujeto. Se realizaron 5 jornadas de CCSN para niños de 2 a 14 años. Acudieron 101 niños, por lo que se logró una cobertura del $82,11 \%$. Se obtuvo adherencia al dispositivo, en un espacio de recreación en el barrio, que a la vez se asoció con hábitos saludables, lo que concretó el sentimiento de responsabilidad familiar respecto a la salud de los niños.

Palabras clave: controles de salud en niños, primer nivel de atención, adherencia.
\end{abstract}

\begin{abstract}
The health team in the San Dionisio Health Center developed a device called Collective Health Checks for Children (CHCC) to minimize the difficulties arise for effective coverage of health checks of children in the neighborhood of Villa Caracol, Bahia Blanca. The objectives of the CHCC were to strengthen the sense of family responsibility for children's health, conduct a screening of children needing urgently health control and strengthen personal living spaces. There were five days of $\mathrm{CHCC}$ for children 2 to 14 year; 101 children were attended, achieving a coverage of $82.11 \%$. Adhesion to the device was achieved in a recreational space in the neighborhood, which in turn was associated with healthy habits, specifying the sense of family responsibility for the health of children.

Key words: Health checks, children, primary care, adherence.
\end{abstract}

http:/ /dx.doi.org/10.5546/aap.2014.271

\section{INTRODUCCIÓN}

En Bahía Blanca, el sistema de salud público está organizado en niveles. Las unidades sanitarias representan el primer nivel de atención, donde se produce la entrada por parte del usuario. Respecto a la atención médica, cuentan con uno o dos médicos de familia según la estructura de la unidad sanitaria (US) respectiva. El segundo nivel de atención, compuesto por hospitales de baja complejidad, cuenta con médicos especialistas en pediatría, ginecología, obstetricia y clínica, que funcionan como línea de apoyo matricial al primer nivel y para la atención de casos que trascienden a este.

La Unidad Sanitaria San Dionisio cuenta con un equipo de salud conformado por una médica especialista en Medicina Familiar, una licenciada en Enfermería, una promotora de salud, dos licenciadas en Psicología y una médica residente de Medicina Familiar.

Esta Unidad Sanitaria registra una población adscrita de 2103 personas. El grupo etario predominante es la franja de 6 a 13 años (Figura 1).

En dicho lugar, se realiza atención médica de baja complejidad, prácticas de enfermería y consultas psicológicas, y fuera de él, el equipo de salud efectúa intervenciones comunitarias, que surgen de los problemas de salud que el equipo de San Dionisio detecta o por demanda repetida de los pacientes-usuarios. Algunas de las intervenciones comunitarias realizadas durante el 
año fueron jornadas para adultos con postas de control de glucemia, registro de presión arterial y peso, consejería, entrega de manzanas y folletería de enfermedades crónicas, sorteo de balanza; charla sobre lavado de manos y prevención del síndrome urémico hemolítico; organización de juegos que desarmaban mitos respecto a la alimentación y sorteo de canasta saludable; taller para madres de armado de juguetes con material reciclable para sus hijos; charla con adolescentes sobre la importancia del deporte, a cargo de jugadores de fútbol de primera división del Club Olimpo; controles de salud colectivos para adolescentes; taller de cocina para la familia; gestión de clases de gimnasia semanales en la Unidad Sanitaria; etc.

Uno de los barrios que compone la población adscrita de la Unidad Sanitaria San Dionisio es Villa Caracol, ubicado a 30 cuadras aproximadamente de la Unidad. Villa Caracol posee una población de 123 niños de 2 a 14 años, de los cuales el 63\% nunca se había realizado un control de salud ni se había vacunado. Presenta los índices de consulta más bajos respecto a otros barrios, sobre todo en relación con los controles de niño sano. Se hace énfasis en este dato porque, de la información analizada sobre el barrio mencionado, fue el más alarmante y, por lo tanto, el puntapié inicial para pensar y desarrollar la experiencia que se describe en el presente texto.

El barrio Villa Caracol tiene 30 años de existencia en la ciudad y se caracteriza por sus condiciones de precariedad y riesgos físicos constantes. Los tipos de viviendas hacen que los límites entre ellas y el espacio público no sean formales. Las condiciones de hacinamiento dejan poco lugar para la intimidad, lo que dificulta o imposibilita la privacidad de los miembros dentro del hogar, como también el juego y el ejercicio físico de los niños.

La forma en que se establecen y desarrollan los lazos, las interacciones o los vínculos interpersonales están condicionados por los entornos materiales de existencia. ${ }^{1}$ Las personas y familias que viven en Villa Caracol están excluidas de las estructuras sociales formales, tales como educación, salud y trabajo, lo que ha provocado a lo largo de los años la generación de modos particulares para sobrevivir o subsistir. Este proceso se ve cristalizado en situaciones puntuales, tales como la ausencia concreta de roles familiares y sociales, y en el desapego y desconocimiento de pautas culturales comunes, que entre otras cosas, tienen que ver con el modo de cuidado de la salud.

Pirámide poblacional. Unidad Sanitaria San Dionisio

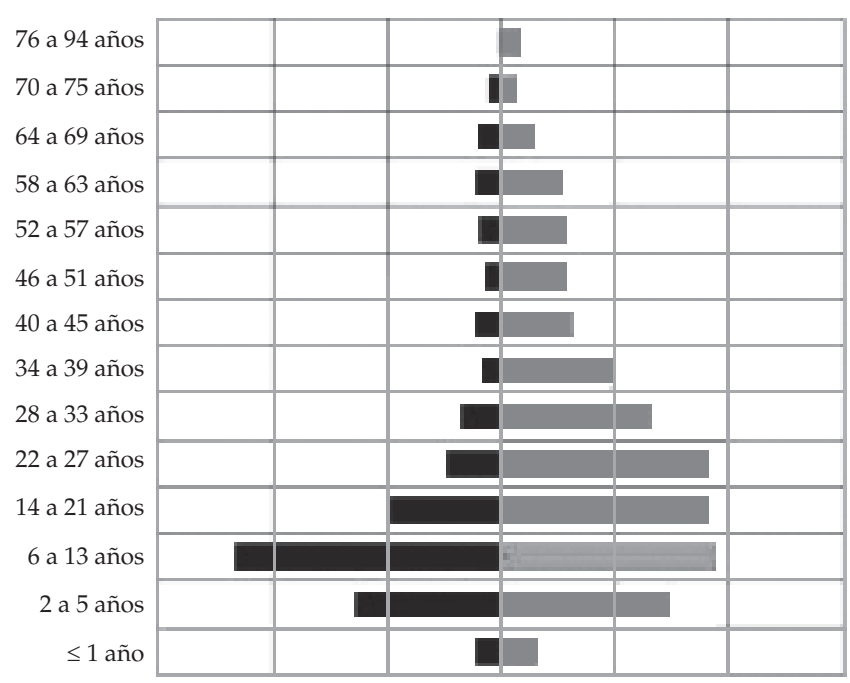

Varones Mujeres 
Los objetivos del dispositivo nombrado fueron:

- Reforzar el sentimiento de responsabilidad familiar respecto a la salud de los niños.

- Realizar un tamizaje de niños que necesitaran un control de salud individual con urgencia.

- Afianzar los espacios de vida personales con los que contaba cada sujeto.

\section{METODOLOGÍA}

El equipo de salud realizó jornadas de CCSN destinadas a niños de 2 a 14 años en los salones de usos múltiples (SUM) del barrio Villa Caracol y de la Unidad Sanitaria San Dionisio. Las últimas dos jornadas se realizaron en el salón de la Unidad Sanitaria para que los habitantes de Villa Caracol, que tienen un vínculo positivo con el equipo de salud que allí trabaja, lo conocieran y supieran que podían contar con él para los controles y otras demandas de salud por su cercanía. Se eligió el grupo de 2 a 14 años en particular, dado que los menores de 2 años necesitan, para evaluar crecimiento y desarrollo, herramientas técnicas y materiales más específicas, que no era posible desarrollar en este dispositivo. Los mayores de 14 años fueron incluidos en controles colectivos para adolescentes, que se llevaron a cabo en el barrio en ocasión diferente.

Las intervenciones elegidas para este dispositivo son las intervenciones definidas obligatorias en un control de salud. ${ }^{2,3}$

Los CCSN se desarrollaron en postas consecutivas, cada una dirigida por un integrante del equipo de salud, con la colaboración de estudiantes de Medicina y Enfermería. Cada posta se planificó y ensayó durante las semanas previas para optimizar el tiempo y coordinar el dispositivo de modo de cumplir los objetivos planteados con responsabilidad y eficiencia. Las postas fueron:

1. Entrevista breve para detectar necesidades subjetivas y recolectar datos inherentes a la historia clínica personal;

2. atención de otras consultas de salud (otitis media aguda, broncoespasmo, realización de recetas de fármacos de uso crónico, faringitis, diarrea aguda, lesiones dérmicas impetiginizadas, tiña, escabiosis, consulta sobre turnos al segundo nivel de atención, etc.);

3. control de vacunación;
4. peso y talla con sus respectivos percentiles (según tablas de percentilo de peso por edad y sexo de OMS y SAP, se consideró bajo peso el percentilo menor de 10);

5. examen de agudeza visual (evaluado con tablas de Snellen a partir de los 4 años);

6. examen odontológico;

7. auscultación cardíaca.

Además de la dimensión material de cada posta, se prestaba especial atención a la etapa del desarrollo madurativo del niño y su modo de vincularse socialmente (según criterios diagnósticos de DSM IV), dado que está demostrado que el bajo nivel socioeconómico de los niños es un problema para su desarrollo madurativo, psicológico y social: si el niño vive en un medio social pobre en estímulos y experiencias, el rendimiento en su desarrollo será bajo en relación con los niños de ambientes más estimulantes. ${ }^{4}$

En paralelo, organizaciones barriales y ONG locales organizaron actividades recreativas, con juegos en modalidad de equipo, en los cuales participaban padres y niños. Al finalizar el pasaje por todas las postas de los CCSN, a cada niño se le entregaba un obsequio, el cual consistía en un envoltorio con cereales, que fue financiado y armado por los integrantes del equipo de salud.

Se convocó a las jornadas mediante información precisa sobre los CCSN, con carteles colocados en el barrio y entrega de invitaciones a domicilios particulares, y se contó con la colaboración de referentes barriales para la difusión.

\section{RESULTADOS}

Se realizaron 5 jornadas. Acudieron 101 niños en total: 42 a la primera jornada, 8 a la segunda, 17 a la tercera, 21 a la cuarta y 13 a la quinta. Se controlaron 14 niños de 2 años, 9 de 3 años, 10 de 4 años, 5 de 5 años, 9 de 6 años, 8 de 7 años, 11 de 8 años, 9 de 9 años, 6 de 10 años, 4 de 11 años, 3 de 12 años, 4 de 13 años y 9 de 14 años. Se logró una cobertura, hasta la fecha, de $82,11 \%$.

Se encontraron 12 niños con bajo peso, 7 con soplos cardíacos, 24 con caries dentales, 12 con disminución de la agudeza visual, 16 con calendario de vacunación incompleto y 9 niños con trastorno disruptivo y déficit de atención. Se resolvieron en acto 25 demandas espontáneas.

En las semanas siguientes, se realizaron intervenciones específicas para abordar los 
problemas de salud detectados: se convocó a una odontóloga y a los niños con caries al SUM para que fueran examinados y se les otorgó un turno en caso de que necesitaran una extracción dental; además, se realizó una fluoración a todos los niños. Se solicitaron turnos para oftalmología en el hospital general para los niños con disminución de la agudeza visual. Se realizaron interconsultas con Pediatría y Cardiología Pediátrica para la evaluación de los niños con soplos cardíacos. Se realizó una jornada de vacunación, en la cual se convocó a los padres con niños que tenían su calendario de vacunación incompleto. A los niños con bajo peso se los convocó a un turno programado en el centro de salud.

\section{COMENTARIO}

Los controles de salud del niño (CSN) son una práctica pertinente y un motivo de consulta frecuente del primer nivel de atención. Al presentarse dificultades para lograr una cobertura efectiva de los CSN en el barrio Villa Caracol de la ciudad de Bahía Blanca, el equipo de salud de la Unidad Sanitaria San Dionisio desarrolló un dispositivo denominado Controles Colectivos de Salud para Niños (CCSN).

Se utiliza la noción de dispositivo porque este, al decir de Foucault, surge como una necesidad para dar respuesta a una demanda, que él denomina Urgencia. ${ }^{5}$ En este caso, entendemos que esa demanda, la urgencia, está relacionada con la baja adherencia que el equipo de salud encontró respecto a los controles de salud de los niños que viven en Villa Caracol, probablemente por desconocimiento de la práctica del control de salud o la frecuencia necesaria.

Se pensó en realizar los CCSN de modo colectivo, dado que conlleva una forma de garantizar un espacio que contrasta con la hegemonía uniprofesional y que incorpora la mirada interdisciplinaria, sin perder la importancia de centrarse en la singularidad de cada niño y su familia. Además, se cuenta con las benevolencias de un espacio cotidiano de encuentro, en donde se puede observar, a modo de evaluación del desarrollo social del niño, cómo interactúa este con sus pares y con los adultos.

Todo proceso de inclusión implica participación y toda participación siempre se refiere a un proceso de inclusión, cualquiera sea su tipo y grado. La participación en sí misma es un acto de ejercicio de poder, que asume diferentes formas y produce distintos efectos según la red de sobredeterminaciones en juego en cada caso. ${ }^{6}$ La salud, como plantea Merhy, es un bien de uso para la persona, porque le representa algo útil que le permite estar en el mundo y poder vivirlo de un modo autodeterminado. ${ }^{7}$

Los CCSN son una respuesta positiva del sistema de salud público, que este propicia y financia, para un problema que hasta el momento no estaba resuelto y que brinda accesibilidad a la población y muestra una dimensión de los niños respecto a su relación vincular con otros niños que no puede evaluarse en un control individual. Se logró adherencia de las familias al dispositivo, que aceptaron formar parte de las jornadas y pusieron en práctica de manera continuada los consejos recibidos en estas. ${ }^{8,9}$ Se concretó el sentimiento de responsabilidad familiar respecto a la salud. Como agregado, se provocó una naturalización de los instrumentos médicos y ya no son de rápido rechazo para los niños.

Una vez finalizada la cobertura de niños respecto a los controles de salud en Villa Caracol, el equipo de la Unidad Sanitaria se ha propuesto generar espacios de formación para sus habitantes acerca de problemas de salud prevalentes del barrio.

Los individuos y los colectivos deben asumir la responsabilidad sobre su proceso de saludenfermedad, pero resulta necesaria también la ampliación de su habilidad para responder a los desafíos de la vida en sociedad. Este tipo de dispositivos intenta superar la desigualdad de poder que predomina en la relación de los profesionales y el usuario, y combina el abordaje promocional con el biomédico. ${ }^{10}$

\section{Agradecimientos}

A los estudiantes de Medicina y Enfermería del Departamento de Ciencias de la Salud de la Universidad Nacional del Sur, que colaboraron en cada jornada con entusiasmo y de modo desinteresado.

Al Méd. (Mg.) Pedro Silberman por los aportes y correcciones.

\section{BIBLIOGRAFÍA}

1. Lomagno C. Cartografía de la micropolítica en el barrio de La Boca. Una experiencia de construcción de demandas por condiciones habitacionales desde la perspectiva de la educación popular y la animación sociocultural. 5. ${ }^{\circ}$ Coloquio Internacional de Animación Sociocultural. 26 a 28 de octubre, Zaragoza, 2011. 
2. Rubinstein A, Terrasa S. Medicina familiar y práctica ambulatoria. 2da ed. Buenos Aires: Panamericana, 2006.

3. Guías de intervención en el primer nivel. Sociedad Uruguaya de Pediatría. [Consulta: 13 de abril de 2013]. Disponible en: www.sup.org.uy/Descarga/control_ periodico.doc.

4. Pando Moreno M, Aranda Beltrán C, Amezcua Sandoval M, Mendoza Roay P, Aldrete Rodríguez M. Desarrollo madurativo del niño en zonas socialmente deprimidas del estado de Michoacán. Investigación en Salud 2003; 5(3): 162-6.

5. Foucault M. Dichos y escritos. Tomo III. Madrid: Nacional, 1994.
6. Ferullo de Parajón A. El triangulo de las tres P: psicología, participación y poder. Buenos Aires: Paidós, 2006.

7. Merhy E. Salud: cartografía del trabajo vivo. Buenos Aires: Lugar Editorial, 2006.

8. Alfonso LM. Acerca del concepto de adherencia terapéutica. Rev Cuba Salud Pública, 2004; 30(4):350-2.

9. Ortiz M, Ortiz E. Psicología de la salud: Una clave para comprender el fenómeno de la adherencia terapéutica. Rev Méd Chile. 2007;135(5):647-52.

10. Carvalho SR. Promoción de la salud, "empowerment" y educación: una reflexión crítica como contribución a la reforma sanitaria. Salud Colectiva 2008;4(3):335-47.

\section{Archivos hace 75 años}

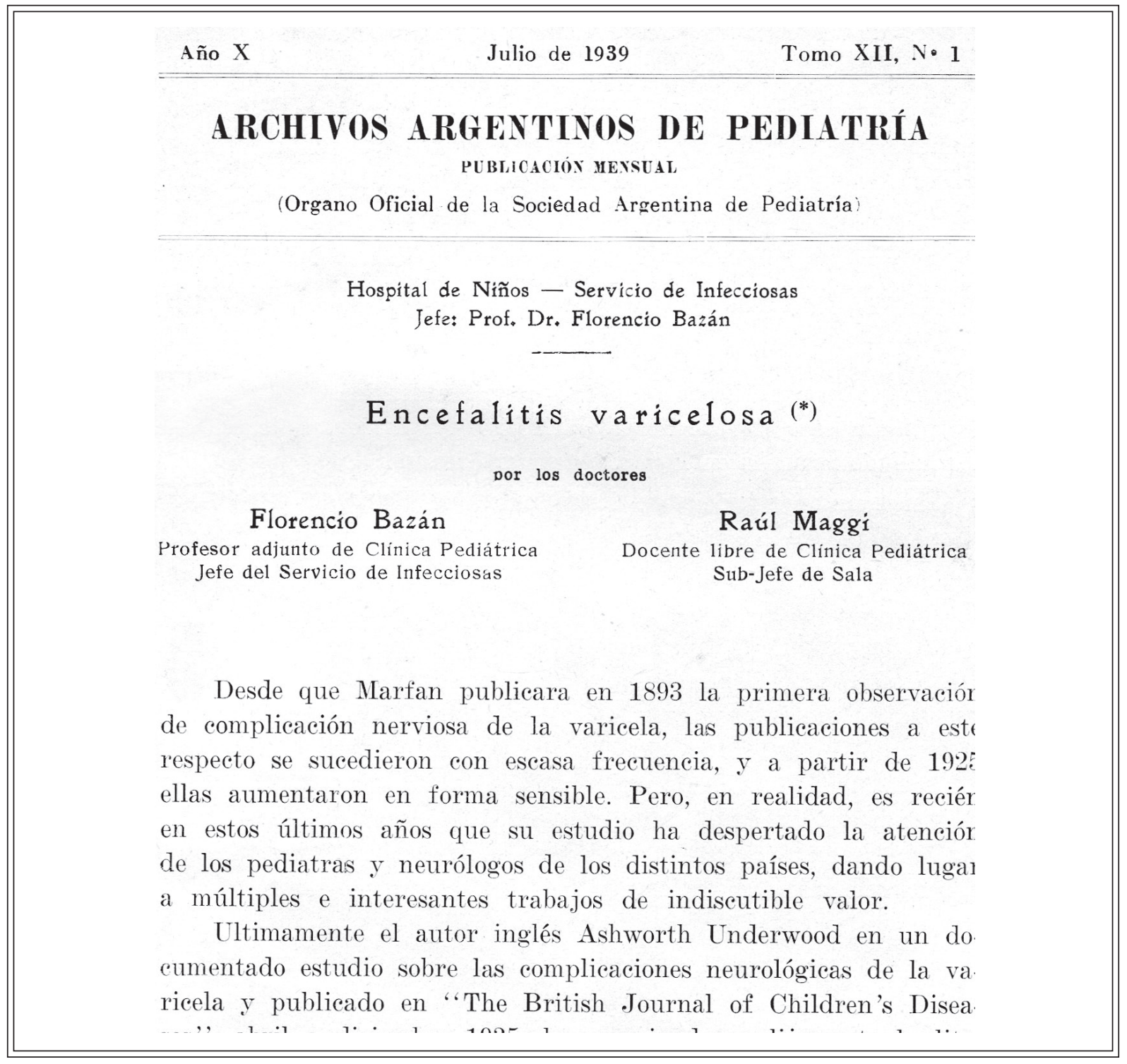

